\title{
Hysterosalpingography findings among women presenting for gynecological imaging in Ado-Ekiti, South western Nigeria
}

\author{
Olufunso S. Aduayi ${ }^{1}$, Ganiyu O. Akanbi ${ }^{1}$, Akinyemi A. Akintayo ${ }^{2}$, Victor A. Aduayi ${ }^{3}$
}

\begin{abstract}
${ }^{1}$ Department of Radiology, Faculty of Clinical Sciences, College of Medicine, Ekiti State University, Ado-Ekiti, Nigeria

${ }^{2}$ Department of Obstetrics and Gynecology, Faculty of Clinical Sciences, College of Medicine, Ekiti State University, Ado-Ekiti, Nigeria

${ }^{3}$ Department of Epidemiology and Community Health, Faculty of Clinical Sciences, College of Medicine, Ekiti State University, Ado-Ekiti, Nigeria
\end{abstract}

Received: 04 April 2016

Accepted: 07 May 2016

\section{*Correspondence: \\ Dr. Olufunso S. Aduayi, \\ E-mail: faduayi@gmail.com}

Copyright: () the author(s), publisher and licensee Medip Academy. This is an open-access article distributed under the terms of the Creative Commons Attribution Non-Commercial License, which permits unrestricted non-commercial use, distribution, and reproduction in any medium, provided the original work is properly cited.

\section{ABSTRACT}

Background: Hysterosalpingography (HSG) is a valuable radiological procedure for imaging the female reproductive tract. This study highlights the spectrum of HSG findings among women presenting for gynaecological imaging in a tertiary health institution in Ado-Ekiti, South western Nigeria.

Methods: A prospective cross sectional study was conducted between October 2014 and September 2015. The study population consisted of 134 women presenting at the Radiology department with request forms for HSG. Pelvic ultrasonography was done using a $3.5 \mathrm{MHz}$ transducer prior to HSG. Imaging findings were documented, results were entered into a spread sheet and analysed using SPSS version 20. Chi square was used to determine association between tubal occlusion, age, type of infertility and previous history of surgery. Statistical significance was set at pvalues $<0.05$.

Results: The age range of the study participants was between 23 to 50 years with a mean age of $34.9 \pm 5.53 y e a r s$. Age group $30-34$ years $(n=43,32.1 \%)$ constituted a majority. Secondary infertility $(n=84,62.7 \%)$ was the most common clinical indication for HSG. The predominant relevant past surgical history was uterine evacuation $(n=41,30.6 \%)$. Normal HSG findings were seen in $34(25.4 \%)$ of the patients while others $(n=100,74.5 \%)$ had various abnormalities, the most common of which were tubal pathologies $(n=89,66.4 \%)$. There was a significant association between age and tubal occlusion $(\mathrm{P}=0.02)$.

Conclusions: HSG is still a relevant gynecological imaging modality in resource limited settings. Tubal occlusion was the most common abnormal HSG finding in this study. There is a need for continuous advocacy on preventive measures targeted at reducing the incidence of tubal pathologies among women in developing countries.

Keywords: Hysterosalpingography, Infertility, Tubal occlusion, Pain

\section{INTRODUCTION}

Hysterosalpingography (HSG) is a radiological procedure that involves the use of contrast media to outline the female reproductive tract. Despite advancements in gynecological imaging in the western world, the ready availability and cost effectiveness of HSG still makes it a key investigation in evaluation of the female genital tract, particularly in the initial diagnostic work up of female infertility in most developing countries. ${ }^{1}$

The primary role of HSG is to evaluate the morphology and the patency of the fallopian tubes. Tubal occlusion is responsible for approximately a third of female 
infertility. ${ }^{2,3}$ and it is the most frequent cause of infertility in Nigeria. ${ }^{4}$ Tubal abnormalities observed with HSG can be congenital, or due to spasm, occlusion or infection. Tubal occlusion manifests as an abrupt cutoff of contrast material with non-opacification of the distal fallopian tube, and can be unilateral or bilateral. Peritubal adhesions prevent contrast material from spilling into the abdominal cavity and distributing freely.,6

HSG also has a role in evaluating the uterine cavity and cervix, as uterine cavity abnormalities can be a contributing cause of subfertility in women. Abnormal uterine findings are reported in as many as $50 \%$ of women with recurrent implantation failure. ${ }^{7}$ These findings include endometrial polyps or fibroids, which are observed as filling defects or uterine wall irregularities on HSG. Intrauterine adhesions and congenital abnormalities can also be demonstrated on HSG. ${ }^{8}$ The purposes of this study was to evaluate all pathologies that were diagnosed by HSG among gynaecologic patients in our centre.

\section{METHODS}

A prospective cross sectional study was conducted in the Radiology department of Ekiti State University Teaching Hospital, Ado-Ekiti, Nigeria between October 2014 and September 2015. Ethical approval was obtained from the institution's Ethics and Research Committee (Protocol Number: EKSUTH/A67/2014/09/005)

A total of 134 women presenting at the Radiology department with request forms for HSG on account of various gynaecological indications were recruited into the study after obtaining informed consent. Patients with suspected pelvic inflammatory disease, or active bleeding per vaginum were excluded from the study. Pelvic ultrasonography was done by radiologists prior to HSG using a $3-5 \mathrm{MHz}$ transducer of an Ultrasound scanner (Mindray real time ultrasound scanner model DCN-2; Shenzhen, China). HSG was done by Radiologists between day 8 and 10 of the menstrual cycle which is within the proliferative phase. This is to prevent inadvertent irradiation of a potential early cyesis and reduce risk of venous intravasation. A scout (preliminary) film was taken first. Intramuscular antispasmodic was administered to minimize cornual spasms. Under aseptic conditions, the cervical os was cannulated and contrast medium was injected retrogradely to outline the cervical canal, uterine cavity and fallopian tubes. Serial contrast films were taken to demonstrate the cervical canal, uterine cavity and fallopian tubes. Delayed films were taken 30 minutes after to complete the imaging series. A static $x$ ray machine was used and digital images were produced with computed radiography. All HSG examinations were interpreted by Radiologists through direct visualization of hard copy images with the aid of a radiograph viewing box. Adjustment of image contrast, brightness and image magnification for optimal views of the fallopian tubes were also done on the computed radiography work station when necessary. Patients with suboptimal images from factors such as contrast media intravasation which makes tubal assessment difficult were also excluded from the study. A normal HSG was defined as one in which the opacified cervical canal and uterine cavity are normal in size with preserved outlines devoid of persistent intracavity filling defects (linear or rounded); both fallopian tubes are opacified, demonstrating a normal calibre and course with bilateral contrast media spillage into the peritoneal cavity and subsequent visualization of uniform smearing of the contrast media within the peritoneal cavity on a 30 minutes delayed film.

Results were entered into a spread sheet and analysed using SPSS version 20. Frequency distributions and percentages were generated. Chi square was used to determine association between tubal occlusion, age, type of infertility and previous abdominal surgery. Statistical significance was set at $\mathrm{p}$-values $<0.05$.

\section{RESULTS}

Table 1: Age distribution.

\begin{tabular}{|lll|}
\hline Age & Frequency $(\mathbf{N}=134)$ & Percentage \\
\hline $20-24$ & 1 & 0.7 \\
$25-29$ & 22 & 16.4 \\
$30-34$ & 43 & 32.1 \\
$35-39$ & 34 & 25.4 \\
$40-44$ & 30 & 22.4 \\
$\geq 45$ & 4 & 3.0 \\
\hline
\end{tabular}

Table 2: Summary of relevant previous surgical history and ultrasonography findings of patients.

\begin{tabular}{|c|c|c|}
\hline Variables & $\begin{array}{l}\text { Frequency } \\
(\mathrm{N}=134)\end{array}$ & Percentage \\
\hline \multicolumn{3}{|l|}{ Previous Surgical history* } \\
\hline Uterine evacuation & 41 & 30.6 \\
\hline Myomectomy & 7 & 5.2 \\
\hline Caesarean section & 5 & 3.7 \\
\hline Salpingectomy & 5 & 3.7 \\
\hline Uterine Adhesiolysis & 1 & 0.7 \\
\hline Nil & 83 & 61.9 \\
\hline \multicolumn{3}{|l|}{ Ultrasound findings } \\
\hline Uterine fibroids only & 34 & 25.4 \\
\hline Small sized uterus & 3 & 2.2 \\
\hline $\begin{array}{l}\text { Uterine fibroids with } \\
\text { ovarian cyst }\end{array}$ & 3 & 2.2 \\
\hline Ovarian mass only & 1 & 1.1 \\
\hline Nabothian cyst & 1 & 1.1 \\
\hline Normal & 92 & 68.7 \\
\hline
\end{tabular}

*multiple responses were recorded in some cases.

A total of 134 women were recruited into the study within a period of one year. The age range was between 23 to 50 years with a mean age of $34.9 \pm 5.53$ years. Women in the age group 30-34yrs $(n=43,32.1 \%)$ constituted the majority of the patients (Table 1). 
Table 3: Hysterosalpingogram findings among study participants.

\begin{tabular}{|lll|}
\hline HisG findings & $\begin{array}{l}\text { Frequency } \\
(\mathrm{N}=134)\end{array}$ & $\begin{array}{c}\text { Percentage } \\
(\%)\end{array}$ \\
\hline $\begin{array}{l}\text { Tubal pathologies } \\
\text { Bilateral Tubal Occlusion }\end{array}$ & 34 & 25.4 \\
Right Tubal Occlusion & 10 & 7.5 \\
Left Tubal Occlusion & 16 & 11.9 \\
Right Hydrosalpinx & 9 & 6.7 \\
Left Hydrosalpinx & 6 & 4.5 \\
Bilateral Hydrosalpinges & 4 & 3.0 \\
Overhanging tubes & 9 & 6.7 \\
Beaded tube & 1 & 0.7 \\
\hline Uterine pathologies & & \\
Uterine Fibroids & 28 & 20.9 \\
Uterine Synaechiae & 11 & 8.2 \\
Congenital Uterine & 4 & 3.0 \\
anomaly* & & \\
Adenomyosis & 2 & 1.3 \\
\hline Cervical pathologies & & \\
Filling defects/irregular & 14 & 10.4 \\
outline & & \\
\hline Peritoneal pathologies & & 17.9 \\
Pelvic Adhesions & 24 & 25.4 \\
\hline Normal findings & 34 & \\
* Arcuate uterus, bicornuate uterus. & \\
\hline
\end{tabular}

* Arcuate uterus, bicornuate uterus.

Table 4: Association between age, type of infertility, previous history of surgery and tubal blockage.

\begin{tabular}{|c|c|c|c|}
\hline Variable & $\begin{array}{l}\text { Bilateral } \\
\text { tubal } \\
\text { blockage }\end{array}$ & $\begin{array}{l}\text { At least one } \\
\text { patent tube }\end{array}$ & $\begin{array}{l}\text { Statistical } \\
\text { indices }\end{array}$ \\
\hline $\begin{array}{l}\text { Age } \\
\leq 35 \\
>35\end{array}$ & $\begin{array}{l}14 \\
20\end{array}$ & $\begin{array}{l}66 \\
34\end{array}$ & $\begin{array}{l}\chi^{2}=9.69 \\
\mathrm{df}=1 \\
\mathrm{p}=0.02 * *\end{array}$ \\
\hline $\begin{array}{l}\text { Type of } \\
\text { infertility } \\
1^{\circ} \\
\text { Infertility } \\
2^{\circ} \\
\text { Infertility }\end{array}$ & $\begin{array}{l}10 \\
23\end{array}$ & $\begin{array}{l}33 \\
61\end{array}$ & $\begin{array}{l}\chi^{2}=5.99 \\
\mathrm{df}=1 \\
\mathrm{p}=0.424\end{array}$ \\
\hline $\begin{array}{l}\text { Previous } \\
\text { surgery* } \\
\text { Yes } \\
\text { No }\end{array}$ & $\begin{array}{l}14 \\
20\end{array}$ & $\begin{array}{l}37 \\
63\end{array}$ & $\begin{array}{l}\chi^{2}=2.24 \\
\mathrm{df}=1 \\
\mathrm{p}=0.524\end{array}$ \\
\hline
\end{tabular}

*Salpingectomy, Myomectomy, Caesarean Section, Uterine Evacuation, Uterine Adhesiolysis $\chi^{2}=$ Pearson Chi-square, **Statistically significant at $\mathrm{p}$ value $<0.05$.

Secondary Infertility $(\mathrm{n}=84,62.7 \%)$ was the most common clinical indication for HSG. Menstrual abnormalities (Amenorrhea, Oligomenorrhoea and Menorrhagia) accounted for $3.7 \%$ while recurrent miscarriages accounted for $1.5 \%$ of clinical indications (Figure 1). The predominant relevant past surgical history (Table 2) among these women was uterine evacuation $(\mathrm{n}=$ $41,30.6 \%$ ) through dilation and curettage either for termination of pregnancy or as part of treatment for spontaneous abortion. Pelvic ultrasonography showed essentially normal findings in $92(68.7 \%)$ of the patients while uterine fibroids was the predominant abnormal ultrasonography finding among the other patients (Table 2).

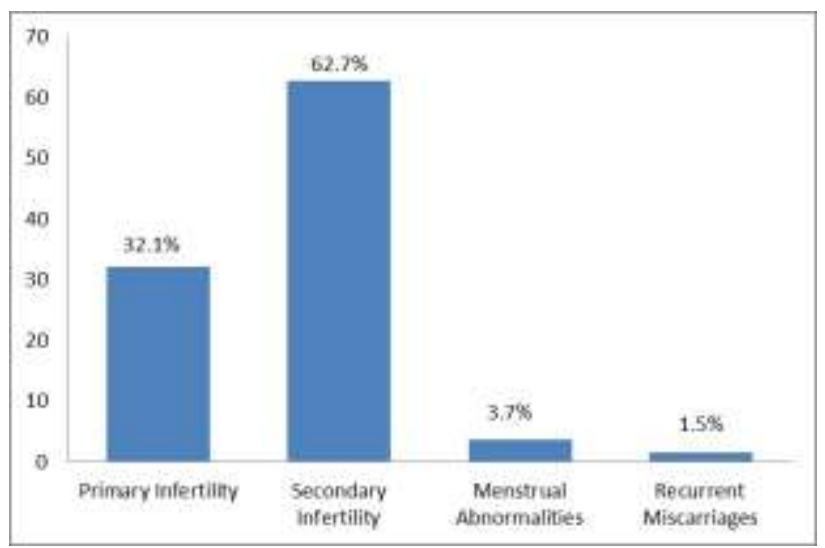

Figure 1: Clinical indications for HSG among patients.

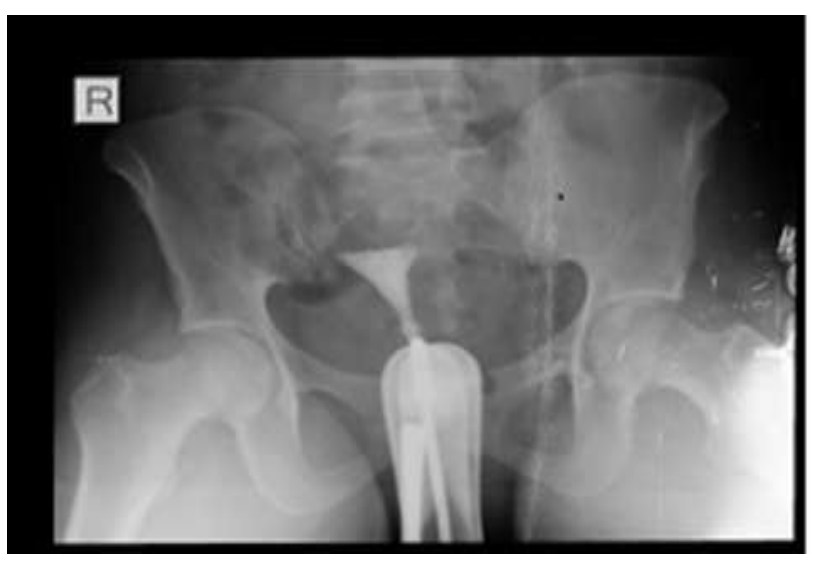

Figure 2: HSG demonstrating bilateral tubal occlusion in a patient with primary infertility.

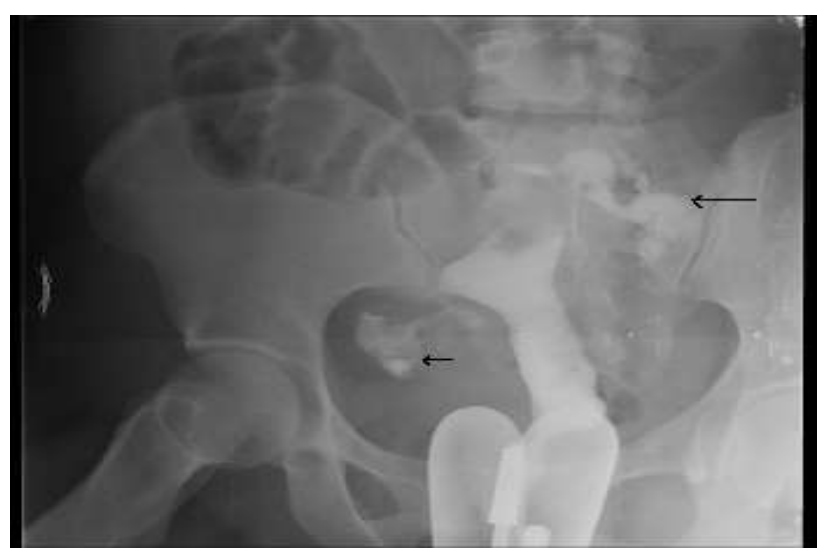

Figure 3: HSG demonstrating bilateral hydrosalpinges (arrows) in a patient with secondary infertility. A contrast filling defect (uterine fibroid) is also seen at the fundal region. 
Only $34(25.4 \%)$ of the patients had normal HSG findings while the remaining $100 \quad(74.5 \%)$ had various abnormalities (Table 3). Tubal pathologies accounted for a significant proportion of the abnormalities, with the most common tubal pathology being tubal occlusion (Figure 2) followed by hydrosalpinges (Figure 3). There was a positive association between the age and tubal occlusion on HSG $(\mathrm{P}=0.02)$ but no significant association was demonstrated between tubal occlusion and type of infertility or relevant previous history of surgery (Table 4).

\section{DISCUSSION}

Infertility was the most common clinical indication for HSG in this study. Majority of the women presented with secondary infertility which is similar to findings obtained from other studies done in Nigeria. ${ }^{1,3,9-13}$ On the contrary a researcher in Thailand reported predominance of primary infertility in their study population. ${ }^{14}$ A greater proportion of the study participants had tubal pathologies as an abnormal imaging finding on HSG and tubal occlusion was the predominant tubal pathology seen. Similar findings were also reported in different parts of Nigeria., ${ }^{3,9,10,12,13}$ Tubal factor accounts for $15-30 \%$ of infertility in all women in developing countries with high rates of pelvic inflammatory disease and limited resources Adetiloye and Danfulani et al reported predominant right tubal occlusion, in contrast to this study which identifies a preponderance of bilateral tubal occlusion in keeping with some other studies. $1,9,12,13,15,16$ Hydrosalpinx was another major tubal abnormality, which was predominantly a unilateral finding(more on the right than left) in this study in keeping with Akinola et al findings but in contrast to other studies that reported predominance of bilateral hydrosalpinges. ${ }^{9,12,17}$ Adetiloye identified increased inflammatory responses on the right side due to the presence of the appendix as a probable cause of predominant right sided hydrosalpinx. Previous unsafe abortion, pelvic inflammatory disease and puerperal sepsis constitute major sources of infection of the female reproductive tract. Hence primary prevention and prompt treatment of infection is crucial to reducing the high incidence of tubal infertility in our environment. ${ }^{12}$

There was a significant association between bilateral tubal occlusion and age $(\mathrm{P}=0.02)$. Age related infertility among women is primarily due to oocyte abnormalities and decreased ovarian reserve. Women tend to have a decline in fertility rate from the age of 35 years and according to a recent study involving 7172 women, older women are more likely to have tubal infertility than younger ones. ${ }^{18}$ This study did not reveal any significant association between tubal occlusion and previous history of abdominal surgery in contrast to findings from another study done by Famurewa et al. ${ }^{19}$ There was also no relationship between bilateral tubal occlusion and type of infertility (primary or secondary).Bilateral tubal occlusion often implies a poor prognosis for possibility of natural conception. It is a major indication for In vitro fertilization (IVF) which is often beyond the financial capacity of most couples trying to achieve conception in a resource poor setting. Some studies have describe the radiographic appearance of the cornual ends when free contrast spillage fails to occur, stating that it is rounded and smooth margined in cases of tubal spasms and pointed in tubal occlusion. ${ }^{3}$ Efforts to minimize cornual spasm during the HSG procedure included giving the patients antispasmodics.

Uterine fibroids constituted the major proportion of uterine pathologies seen, in keeping with other studies. ${ }^{9,13,17,20}$ It was observed that cases of multiple uterine fibroids often require extra volume of contrast media during HSG procedures and this may not be unconnected with the bulky nature of their uterus. Fibroid nodules show as abnormal uterine cavity outlines and filling defects on HSG. Congenital uterine anomalies were demonstrated in 3\% of the Hysterosalpingograms in this study. The most common congenital anomaly that was encountered was arcuate uterus. This corroborates the fact that Nigeria falls within the infertility belt with a majority of the factors responsible for infertility being acquired rather than congenital, Other less commonly encountered HSG abnormalities in this study included two cases of suspected adenomyosis appearing as irregular branching outpouchings of contrast radiating from the uterine cavity, representing extension of the endometrial glands into the myometrium. ${ }^{18} \mathrm{~A}$ case of beaded appearance of the fallopian tube in keeping with salpingitis isthmica nodosa was also encountered, similar to Akinola et al findings. ${ }^{9}$

Despite technological advancements in the evaluation of gynaecological pathologies HSG still plays a vital role in gynaecological imaging in resource limited settings. In most centres in developing nations, ultrasonography and HSG are available for baseline gynaecological imaging while more sophisticated equipment's such as laparoscope; hysteroscope and MRI are expensive and not readily available. Both pelvic ultrasonography and HSG were done for our patients and this provided a better radiological appraisal of the female reproductive tract. Pelvic ultrasonography defined the uterine contours, evaluated for uterine fibroids and other myometrial, endometrial and adnexal abnormalities. The pouch of douglas was also visualized for presence of significant free fluid which may serve as a pointer to pelvic inflammatory disease (PID). The 10 day rule regarding procedures involving radiation exposure in women was adhered to; hence HSG was done between day 8 and 10 of the menstrual cycle. The isthmus of the fallopian tube is most distensible at this time and this facilitates easy demonstration of the tubes when filled with contrast medium. $^{21}$ The second half of the menstrual cycle is often avoided because of inadvertent irradiation of a potential early pregnancy and because the thickened secretory endometrium increases the risk of venous intravasation which makes proper assessment of the fallopian tubes 
difficult. ${ }^{1}$ Ideally HSG is done under fluoroscopic guidance. However due to non-availability of a fluoroscopy machine at the centre as at the time the study was being conducted, a static $\mathrm{x}$ ray machine was utilized and digital images were produced using computed radiography. This offered better image quality as opposed to regular analogue films which is still being used in most centres in other parts of Nigeria. HSG is a simple safe and cost effective radiographic study of the uterine cavity and the fallopian tube with $65 \%$ sensitivity and $83 \%$ specificity for detecting tubal blockage. ${ }^{22}$ Some studies have reported that Laparoscopy and dye test is more informative. ${ }^{6,23,24}$ Laparoscopy may be omitted in women with normal HSG or suspected unilateral distal tubal pathology on HSG, since study has not shown it to change the original treatment plan indicated by HSG in $95 \%$ of the patients. ${ }^{24}$ However, laparoscopy should be recommended in cases with suspected bilateral tubal occlusion on HSG, since in one study, it altered the original treatment plan in $30 \%$ of the patients from invitro fertilization (IVF) to induction of ovulation with intra uterine insemination (IUI). ${ }^{24}$

HSG has limitations such as exposure of patients to ionising radiation, invasive nature of the procedure, inability to define uterine contours, attendant complications such as severe pain, haemorrhage, pelvic infection and syncopy. ${ }^{3}$ The only complication experienced by virtually all the patients in this study was pain during the procedure. Procedural pain during HSG was mostly experienced while grasping the lip of the cervix with a forceps, fixing the cannula, and during contrast media spillage through the fallopian tubes into the peritoneal cavity. Some clients have described the pain experienced during the contrast spillage as severe abdominal cramps likened to a worse form of pain experienced during dysmenorrhea. Mild analgesics are usually prescribed for pain relief.

\section{CONCLUSION}

HSG plays a key role in gynaecological imaging in resource limited settings. Tubal pathologies were the most common abnormal imaging finding on HSG, of which tubal occlusion was predominant and found to have a significant association with age. There is a need for continuous advocacy on preventive measures targeted at reducing the incidence of tubal pathologies among women in developing countries.

\section{Funding: No funding sources}

Conflict of interest: None declared

Ethical approval: The study was approved by the Institutional Ethics Committee

\section{REFERENCES}

1. Bukar M, Mustapha Z, Takai U, Tahir A. Hysterosalpingographic findings in infertile women:
A seven year review. Nigerian journal of clinical practice. 2011;14(2):168-70.

2. Miller JH, Weinberg RK, Canino NL, Klein NA, Soules MR. The pattern of infertility diagnoses in women of advanced reproductive age. American journal of obstetrics and gynecology. 1999;181(4):952-7.

3. Bello T. Pattern of tubal pathology in infertile women on hysterosalpingography in Ilorin, Nigeria. 2004.

4. Audu B, Massa A, Bukar M, El-Nafaty A, Sa'ad S. Prevalence of utero-tubal infertility. Journal of obstetrics and gynaecology. 2009;29(4):326-8.

5. Simpson Jr WL, Beitia LG, Mester J. Hysterosalpingography: A Reemerging Study 1. Radiographics. 2006;26(2):419-31.

6. Mol BW, Collins JA, Burrows EA, Van der Veen F, Bossuyt PM. Comparison of hysterosalpingography and laparoscopy in predicting fertility outcome. Human Reproduction. 1999;14(5):1237-42.

7. Brown SE, Coddington CC, Schnorr J, Toner JP, Gibbons W, Oehninger S. Evaluation of outpatient hysteroscopy, saline infusion hysterosonography, and hysterosalpingography in infertile women: a prospective, randomized study. Fertility and sterility. 2000;74(5):1029-34.

8. Pundir J, El Toukhy T. Uterine cavity assessment prior to IVF. Women's Health. 2010;6(6):841-8.

9. Akinola R, Akinola O, Fabamwo A. Infertility in women: Hysterosalpingographic assessment of the fallopian tubes in Lagos, Nigeria. Educational Research and Reviews. 2009;4(3):86.

10. Okafor C, Okafor C, Okpala O, Umeh E. The pattern of hysterosalpingographic findings in women being investigated for infertility in Nnewi, Nigeria. Nigerian journal of clinical practice. 2010;13(3).

11. Ibekwe P, Udensi M, Imo A. Hysterosalpingographic findings in patients with infertility in South eastern Nigeria. Nigerian Journal of Medicine. 2010;19(2).

12. Danfulani M, Mohammed MS, Ahmed SS, Haruna YG. Hysterosalphingographic findings in women with infertility in Sokoto North Western Nigeria. African Journal of Medical and Health Sciences. 2014;13(1):19.

13. Mgbor S. Pattern of Hysterosalpingographic findings in gynaecological patients in Enugu. Nig Med J. 2006;47:14-6.

14. Sinawat S, Pattamadilok J, Seejorn K. Tubal abnormalities in Thai infertile females. J Med Assoc Thai. 2005;88(6):723-7.

15. Hoffman L, Chan K, Smith B, Okolo S. The value of saline salpingosonography as a surrogate test of tubal patency in low-resource settings. International journal of fertility and women's medicine. 2004;50(3):135-9.

16. 16. Adetiloye V. Hysterosalpingography in investigation of infertility: experience with 248 patients. West African journal of medicine. 1992;12(4):191-6. 
17. Nwankwo N, Akani C. Pattern of hysterosalpingographic findings in infertility in Port harcourt. West African J Radio. 2005;12:15-9.

18. Maheshwari A, Hamilton M, Bhattacharya S. Effect of female age on the diagnostic categories of infertility. Human Reproduction. 2008;23(3):538-42.

19. Famurewa O, Adeyemi A, Ibitoye O, Ogunsemoyin O. Association between history of abdominopelvic surgery and tubal pathology. African health sciences. 2013;13(2):441-6.

20. Kiridi E, Ibrahim I, Lawani L. Hysterosalpingography: Still relevant in the evaluation of infertility in the Niger Delta. International Journal of Medicine and Biomedical Research. 2015;4(1):50-4.
21. Ronald G. The female reproductive systems in: Grainger RG, Allison DJ, editors. Diagnostic radiology.3:1809-69.

22. Khalaf Y. Tubal subfertility. Bmj. 2003;327(7415):610-3.

23. Idrisa A, Ojiyi E, Hamidu A. Hysterosalpingography versus Laparoscopy in the Evaluation of Female Infertility in Maiduguri, Nigeria. Tropical Journal of Obstetrics and Gynaecology. 2004;20(1):20-3.

24. Lavy Y, Lev-Sagie A, Holtzer H, Revel A, Hurwitz A. Should laparoscopy be a mandatory component of the infertility evaluation in infertile women with normal hysterosalpingogram or suspected unilateral distal tubal pathology? European Journal of Obstetrics and Gynecology and Reproductive Biology. 2004;114(1):64-8.

Cite this article as: Aduayi OS, Akanbi GO, Akintayo AA, Aduayi VA. Hysterosalpingography findings among women presenting for gynecological imaging in Ado-Ekiti, South western Nigeria. Int J Reprod Contracept Obstet Gynecol 2016;5:1906-11. 